Proc. Indian Acad. Sci. (Earth Planet. Sci.), Vol. 93, No. 2, July 1984, pp. 91-103.

(C) Printed in India.

\title{
Ozone in the mesosphere and lower thermosphere
}

\author{
L S HINGANE \\ Indian Institute of Tropical Meteorology, Pune 411005, India
}

MS received 21 December 1981; revised 28 February 1984

\begin{abstract}
Ahstract. One-dimensional photochemical diffusive model has been used to obtain the altitude and temporal variation of $\mathrm{O}_{3}$ in the range of 50 to $150 \mathrm{~km}$ beight. More than 60 chemical reactions and photo-dissociatons of oxygen-hydrogen atmosphere are incorporated. Their contribution in the formation or distribution directly or indirectly, is critically examined. In the altitude profile of $\mathrm{O}_{3}$ concentration, it is observed that there is slight increase in concentration around $85 \mathrm{~km}$ which is due to the increase in production rate at that height through the following reaction as: $\mathrm{O}+\mathrm{O}_{2}+\mathrm{M} \rightarrow \mathrm{O}_{3}+\mathrm{M}(\mathrm{M}=$ total atmospheric concentration) and the sharp decrease above $90 \mathrm{~km}$ is also through the same reaction. Two altitude profiles of ozone concentration, one from photochemical equilibrium and other from nonequilibrium model are obtained. It is seen that in the mesosphere these two profiles are comparable. Further it is also seen that the average night time concentration is more than that in the day. Results obtained are compared with experimental values.
\end{abstract}

Keywords. Ozone concentration; mesosphere; oxygen-hydrogen atmosphere; diffusion.

\section{Introduction}

Ozone in the atmosphere acts as a biological shield for the earth's biosphere because it absorbs almost all penetrating uv solar radiation harmful to lives on earth. Chapman (1930) suggested the existence of $\mathrm{O}_{3}$ in the earth's atmosphere as a result of photodissociation of molecular oxygen and subsequent recombination of atomic and molecular oxygen in the presence of the third body. The $\mathrm{O}_{3}$ in turn is destroyed by photodissociation and recombination with atomic oxygen.

The distribution of ozone in the middle atmosphere can be obtained from a static model as its chemical lifetime in this altitude range is smaller than that of the mixing time. Below $40 \mathrm{~km}$ equilibrium theory is not applicable because as the altitude decreases, the chemical lifetime of $\mathrm{O}_{3}$ increases and the vertical as well as horizontal diffusion becomes important. However, other constituents involved in the production and loss mechanism of $\mathrm{O}_{3}$ directly or indirectly have longer chemical lifetime than mixing time or diffusive time (e.g. $\mathrm{O}_{2}, \mathrm{O}\left({ }^{3} \mathrm{P}\right), \mathrm{H}$ etc.). For them it is necessary to include the transport term in the model. Studies based on the dynamic model were started during the beginning of this decade (Shimazaki and Laird 1970, 1972; Hunt 1971, 1973; Thomas and Bowman 1972; Crutzen 1974; Shimazaki and Ogawa 1974; Fukuyama 1974). This paper presents more detailed aspect of chemical behaviour. Onedimensional photochemical diffusive model is developed and employed to study the chemical behaviour as well as altitude and temporal variation of $\mathrm{O}_{3}$ in the mesosphere and lower thermosphere. Numerical technique used for the solution of the set of nonlinear differential equations is also presented. 


\section{Dynamic model}

\subsection{Theoretical formulation}

Any molecule can in principle move from one point to another and while doing so, will carry with it its mass, velocity, and internal energy. This movement is mainly controlled by eddy diffusion, molecular diffusion, meridional circulation, planetary waves, solar radiation, and chemical sources and sinks. Therefore, concentration of any constituent in the atmosphere is controlled by the above parameters. Because of the strong meridional circulation and planetary waves in the lower atmosphere, horizontal gradient of the density of the minor constituent is strong and only three-dimensional (3-D) or to some extent two-dimensional (2-D) model can take care of this horizontal and vertical gradients. However, as altitude increases, the horizontal gradient decreases and above $50 \mathrm{~km}$ it is not comparable with the vertical gradient therefore, 1-D model governed by eddy, molecular diffusion as well as photochemical, chemical production and loss terms can give reasonable result in aeronomy with sufficient degree of accuracy. Accordingly we have designed the $1-D$ photochemical diffusion model for the present work in the region 50 to $150 \mathrm{~km}$. The general form of the equations is as follows;

$$
\begin{aligned}
\frac{\partial Y_{j}(z, t)}{\partial t}= & P_{j}(z, t)-Q_{j}(z, t) \cdot Y_{j}(z, t) \\
& -\frac{\partial}{\partial z}\left\{[ Y _ { j } ] \left[\left(\frac{1}{\left[Y_{j}\right]} \cdot \frac{\partial Y_{j}}{\partial z}+\frac{1}{T} \cdot \frac{\partial T}{\partial z}\right)(K+E)\right.\right. \\
& \left.\left.+\left(\frac{K}{H_{a}}+\frac{E}{H_{j}}\right)\right]\right\} .
\end{aligned}
$$

Here, $Y_{j}(z, t)$ represents the concentration of the $j$ th constituent at height $z \mathrm{~km}$ above the ground and at time $t$. In our model $j$ takes values from 1 to 13.T, D,E, $H_{j}$ and $H_{a}$ denote respectively the atmospheric temperature, molecular diffusion coefficient, eddy diffusion coefficient, scale heights of $j$ th constituent and average scale height. It is not possible to list all the 13 constituents with their chemical and photochemical production $P$ and destruction $Q$. However, as an example the production and destruction terms for atomic oxygen and atomic hydrogen are given as:

$$
\begin{aligned}
P_{o}(z, t)= & J_{1}\left[\mathrm{O}_{2}\right]+2 J_{2}\left[\mathrm{O}_{2}\right]+J_{3}\left[\mathrm{O}_{3}\right]+J_{4}\left[\mathrm{O}_{3}\right] K_{16}[\mathrm{OH}][\mathrm{OH}] \\
& +K_{9}\left[\mathrm{O}_{2}\right]\left[\mathrm{O},{ }^{1} \mathrm{D}\right]+K_{48}[\mathrm{H}][\mathrm{OH}]+K_{42}[\mathrm{H}]\left[\mathrm{O}_{2}\right]+\text { etc., } \\
Q_{o}(z, t)= & K_{2}\left[\mathrm{O}_{2}\right][\mathrm{M}]+K_{3}\left[\mathrm{O}_{3}\right]+K_{21}[\mathrm{OH}]+K_{22}\left[\mathrm{HO}_{2}\right] \\
& +K_{33}\left[\mathrm{H}_{2} \mathrm{O}_{2}\right]+K_{35}\left[\mathrm{H}_{2}\right]+\text { etc., } \\
P_{H}(z, t)= & K_{21}[\mathrm{O}][\mathrm{OH}]+J_{5}\left[\mathrm{H}_{2} \mathrm{O}\right]+K_{37}\left[\mathrm{H}_{2}\right][\mathrm{OH}] \\
& +K_{36}\left[\mathrm{H}_{2}\right]\left[\mathrm{O},{ }^{1} \mathrm{D}\right]+K_{35}\left[\mathrm{H}_{2}\right][\mathrm{O}], \\
Q_{H}(z, t)= & K_{20}\left[\mathrm{O}_{3}\right]+K_{23}\left[\mathrm{O}_{2}\right][\mathrm{M}]+\mathrm{H}_{29}\left[\mathrm{HO}_{2}\right] \\
& +K_{30}[\mathrm{OH}]+K_{34}\left[\mathrm{H}_{2} \mathrm{O}_{2}\right]
\end{aligned}
$$

where the rectangular bracket represents the concentration of the respective constituent, $J_{i}$ are the photodissociation rate coefficients which are the functions of height 
and time; and $K_{i}$ the reaction rate coefficients. Equation (1) is a set of nonlinear, second order, couple partial differentials for 13 constituents and can be solved by a specially developed numerical scheme only.

Above $50 \mathrm{~km}$ altitude concentration of $\mathrm{O}_{3}$ is obtained from its photochemical equilibrium model. However, as mentioned earlier ozone cannot be studied independently without considering other constituents which influence its distribution. We therefore, developed simultaneous partial differential equations for 13 constituents viz $\mathrm{O}_{3}, \mathrm{O}_{2}, \mathrm{O}_{2}\left({ }^{1} \Delta_{9}\right), \mathrm{O}\left({ }^{3} \mathrm{P}\right), \mathrm{O}\left({ }^{1} \mathrm{D}\right), \mathrm{O}\left({ }^{1} \mathrm{~S}\right), \mathrm{H}, \mathrm{OH}, \mathrm{OH}^{*}, \mathrm{H}_{2} \mathrm{O}, \mathrm{HO}_{2}, \mathrm{H}_{2} \mathrm{O}_{2}, \mathrm{H}_{2}$. Of these, constituents like $\mathrm{O}_{2}, \mathrm{O}\left({ }^{3} \mathrm{P}\right), \mathrm{H}, \mathrm{H}_{2} \mathrm{O}, \mathrm{H}_{2} \mathrm{O}_{2}$, have longer chemical lifetime and hence only for these the transport term arising due to eddy or molecular diffusion is included. The chemical lifetime is obtained from:

$$
\tau=\left[N_{i}\right] /\left[Q_{i}, N_{i}\right]
$$

where $Q_{i}$ is the rate of destruction, and $N_{i}$ the density of the $i$ th species.

\subsection{Photodissociation}

Photochemical and photolytic reactions are listed in table 1 . The photodissociation rate coefficient is computed using the following formula:

$$
\begin{aligned}
J_{i}(z)= & \sum Q_{i}(\lambda) \cdot \sigma(\lambda), L_{\tau} \exp \left[-\sum_{\lambda} \sigma(\lambda)\right] \\
& \times\left[N_{i}(z)\right] \times\left[(1+z / R)\left(1+2 z / R-\cos ^{2} \phi\right)^{-1 / 2}\right],
\end{aligned}
$$

where $\cos \phi=\cos \theta \cos \Lambda \cos \delta+\sin \Lambda \sin \delta$ and $J_{i}(z)$ is the photodissociation rate of ith constituent at height $z ; Q_{i}(\lambda)$ the quantum efficiency of $i$ th constituent at wavelength $\lambda ; \sigma(\lambda)$ the absorption cross-section of $i$ th constituent at wavelength $\lambda ; L_{T}$ the solar photon flux (no. of photon/ $\mathrm{cm}^{2}$ at the top of the atmosphere); $N_{i}(z)$ the number density of $i$ th constituent at height $z ; R$ the radius of the earth; $\Lambda$ the latitude of the earth; $\delta$ the solar declination angle; $\theta$ the zenith angle. Solar photon flux data and absorption cross-section data for $\mathrm{O}_{3}$ and $\mathrm{O}_{2}$ in the corresponding wavelength region have been taken mainly from Ackerman (1971) and Ackerman et al (1970).

\subsection{Diffusion}

In the mesosphere temperature decreases with altitude up to mesopause. Therefore the vertical convection should be greater than in the thermosphere where temperature increases with altitude. However, in the 85 to $110 \mathrm{~km}$ region, there is strong vertical shear in the horizontal wind induced by the upward propagating gravity waves and atmospheric tides and hence eddy diffusion increases up to the turbopause (at $110 \mathrm{~km}$ ) above which molecular diffusion dominates the eddy diffusion. In fact there is no satisfactory way of determining accurately the eddy diffusion coefficient value. The eddy diffusion coefficient values adopted in our model are shown in figure 1. For molecular diffusion coefficient we have followed Colegrove et al (1965) 
Table 1(a). Chemical reactions incorporated in the present model.

\begin{tabular}{|c|c|c|}
\hline No. & Reactions and references & Rate coefficient \\
\hline $\boldsymbol{R}_{2}$ & $\begin{array}{l}\mathrm{O}+\mathrm{O}+\mathrm{M} \rightarrow \mathrm{O}_{2}+\mathrm{M} \\
\text { Gattinger (1971) }\end{array}$ & $K_{1}=(2.87 \pm 0.3) \times 10^{-34} \exp (710 \pm 175 / \mathrm{T})$ \\
\hline$R_{2}$ & $\begin{array}{l}\mathrm{O}+\mathrm{O}_{2}+\mathrm{M} \rightarrow \mathrm{O}_{3}+\mathrm{M} \\
\text { Hue et al }(1972) \text {, NBSIR (1974). }\end{array}$ & $\begin{array}{l}K_{20}=1.0 \times 10^{-34} \exp (104 / R T) \\
K_{2 b}=1.1 \times 10^{-34} \exp (500 / \mathrm{T}) \text { (for strato- } \\
\text { sphere) }\end{array}$ \\
\hline $\boldsymbol{R}_{\mathbf{3}}$ & $\begin{array}{l}\mathrm{O}+\mathrm{O}_{3} \rightarrow 2 \mathrm{O}_{2} \\
\text { Simonaitis and Heikoklen (1973) }\end{array}$ & $K_{3}=1.3 \times 10^{-11} \exp (-2200 / \mathrm{T})$ \\
\hline $\boldsymbol{R}_{4}$ & $\begin{array}{l}\mathrm{O}+\mathrm{O}+\mathrm{O} \rightarrow \mathrm{OS}+\mathrm{O}_{2} \\
\text { Felder and Young }(1972)\end{array}$ & $K_{4}=4.8 \times 10^{-33}$ \\
\hline $\boldsymbol{R}_{\mathbf{s}}$ & $\begin{array}{l}O\left({ }^{1} S\right) \rightarrow O^{1} D+h v \\
\text { Zipf }(1969)\end{array}$ & $K_{s}=1.4 \times 10^{0}$ \\
\hline$R_{6}$ & $\begin{array}{l}\mathrm{O}\left({ }^{1} \mathrm{~S}\right)+\mathrm{O}_{2} \rightarrow \mathrm{O}+\mathrm{O}_{2} \\
\text { Atkinson and Welge (1972) }\end{array}$ & $K_{6}=4.9 \times 10^{-12} \exp (-850 \pm 100)$ \\
\hline $\boldsymbol{R}_{7}$ & $\begin{array}{l}O\left({ }^{\prime} D\right)+M \rightarrow O+M \\
\text { Fukuyama }(1974)\end{array}$ & $K_{7}=5 \times 10^{-11}$ \\
\hline $\boldsymbol{R}_{\mathbf{8}}$ & $\begin{array}{l}\mathrm{O}\left({ }^{1} \mathrm{D}\right) \rightarrow \mathrm{O}+h v \\
\text { Zipf }(1969)\end{array}$ & $K_{8}=1 \times 10^{-2}$ \\
\hline $\boldsymbol{R}_{\mathbf{g}}$ & $\begin{array}{l}O\left({ }^{1} D\right)+O_{2} \rightarrow O_{2}+O \\
\text { Zipf (1969) }\end{array}$ & $K_{9}=5 \times 10^{-11}$ \\
\hline $\begin{array}{l}R_{10} \\
R_{11}\end{array}$ & $\begin{array}{l}\mathrm{O}_{2}\left({ }^{1} \Delta_{9}\right)+\mathrm{O}_{3} \rightarrow 2 \mathrm{O}_{2}+\mathrm{O} \\
\mathrm{O}_{2}\left({ }^{1} \Delta_{9}\right) \rightarrow \mathrm{O}_{2}+h y \\
\text { Wallace and Hunten (1968) }\end{array}$ & $\begin{array}{l}K_{10}=2.5 \times 10^{-11} \\
K_{11}=8.5 \times 10^{-2}\end{array}$ \\
\hline$R_{12}$ & $\begin{array}{l}\mathrm{O}_{2}\left({ }^{1} \Delta_{9}\right) \rightarrow \mathrm{O}_{2}+h v \\
\text { Evans et al }(1968)\end{array}$ & $K_{12}=2.8 \times 10^{-4}$ \\
\hline$R_{13}$ & $\begin{array}{l}\left({ }^{1} \Delta_{9}\right)+M \rightarrow O_{2}+M \\
\text { Beaker }(1971)\end{array}$ & $K_{13}=10^{-20}$ \\
\hline$R_{14}$ & $\begin{array}{l}\mathrm{O}_{2}\left({ }^{1} \Delta_{9}\right)+\mathrm{O}_{3} \rightarrow 2 \mathrm{O}_{2}+\mathrm{O} \\
\text { Findley and Snelling }(1971)\end{array}$ & $K_{14}=3.2 \times 10^{-13}$ \\
\hline$R_{13}$ & $\begin{array}{l}\mathrm{O}_{2}\left({ }^{1} \mathrm{D}\right)+\mathrm{O} \rightarrow \mathrm{O}_{2}+\mathrm{O} \\
\text { Clark and Wayne }(1969)\end{array}$ & $K_{15}=3 \times 10^{-11}$ \\
\hline$R_{16}$ & $\begin{array}{l}\mathrm{O}\left({ }^{1} \mathrm{D}\right)+\mathrm{H}_{2} \mathrm{O} \rightarrow 2 \mathrm{OH} \\
\text { Biedenkapp et al }(1970)\end{array}$ & $K_{16}=3 \times 10^{-11}$ \\
\hline$R_{17}$ & $\begin{array}{l}\mathrm{O}\left({ }^{1} \mathrm{~S}\right)+\mathrm{H}_{2} \mathrm{O} \rightarrow 2 \mathrm{OH} \\
\text { Filseth et al }(1970)\end{array}$ & $K_{17}=7 \pm 3.5 \times 10^{-11}$ \\
\hline $\boldsymbol{R}_{\mathbf{1 8}}$ & $\begin{array}{l}\mathrm{O}_{2}\left({ }^{t} \Delta_{9}\right)+\mathrm{O}_{2} \rightarrow 2 \mathrm{O}_{2} \\
\text { Findley and Snelling }(1971)\end{array}$ & $K_{18}=2.2 \times 10^{-18}$ \\
\hline $\boldsymbol{R}_{19}$ & $\begin{array}{l}O\left({ }^{1} \mathrm{~S}\right)+O\left({ }^{3} \mathrm{P}\right) \rightarrow 20 \\
\text { Felder and Young }(1972)\end{array}$ & $K_{19}=7.5 \times 10^{-12}$ \\
\hline $\boldsymbol{R}_{20}$ & $\begin{array}{l}\mathrm{H}+\mathrm{O}_{3} \rightarrow \mathrm{OH}^{*}+\mathrm{O}_{2} \\
\text { Koufman }(1964) \text {, Nicolet (1971), Gattinger } \\
\text { (1971). }\end{array}$ & $\begin{array}{l}K_{20 a}=2.6 \times 10^{-11} \\
K_{20 b}=1.5 \times 10^{-12} \\
K_{20 c}=1.5 \times 10^{-11} \exp (-200 / \mathrm{T})\end{array}$ \\
\hline$R_{21}$ & $\begin{array}{l}\mathrm{OH}+\mathrm{O} \rightarrow \mathrm{H}+\mathrm{O}_{2} \\
\text { Koufman }(1965) \text { and Lloyd et al (1972). }\end{array}$ & $\begin{array}{l}K_{21 a}=5.0 \times 10^{-11} \\
K_{21 b}=1.8 \times 10^{-12}\end{array}$ \\
\hline$R_{22}$ & $\begin{array}{l}\mathrm{HO}_{2}+\mathrm{O} \rightarrow \mathrm{OH}+\mathrm{O}_{2} \\
\text { Nicolet }(1970)\end{array}$ & $K_{22}=3 \times 19^{-12}(\mathrm{~T})^{1 / 2}$ \\
\hline$R_{23}$ & $\begin{array}{l}\mathrm{H}+\mathrm{O} 2+\mathrm{M} \rightarrow \mathrm{HO} 2+\mathrm{M} \\
\text { Kurylo }(1972)\end{array}$ & $K_{23}=1.9 \times 10^{-32} \exp (236 / \mathrm{T})$ \\
\hline$R_{24}$ & $\begin{array}{l}\mathrm{OH}+\mathrm{O}_{2} \rightarrow \mathrm{HO}_{2}+\mathrm{O}_{2} \\
\text { Anderson and Koufman (1973) }\end{array}$ & $K_{24}=1.3 \times 10^{-12} \exp (-950 / \mathrm{T})$ \\
\hline$R_{25}$ & $\begin{array}{l}\mathrm{HO}_{2}+\mathrm{O}_{3} \rightarrow \mathrm{OH}+2 \mathrm{O}_{2} \\
\text { Simonaitis and Heicklen (1973) }\end{array}$ & $K_{25}=3.3 \times 10^{-14} \exp (-1000 / T)$ \\
\hline$R_{26}$ & $\begin{array}{l}\mathrm{OH}+\mathrm{OH} \rightarrow \mathrm{H}_{2} \mathrm{O}+\mathrm{O} \\
\text { Whitten et al }(1973)\end{array}$ & $K_{26}=1 \times 10^{-11} \exp (-550 / \mathrm{T})$ \\
\hline
\end{tabular}


Taule 1(a). (contd.)

\begin{tabular}{|c|c|c|}
\hline No. & Reactions and references & Rate coefficient \\
\hline $\boldsymbol{R}_{\mathbf{2 1}}$ & $\begin{array}{l}\mathrm{HO}_{2}+\mathrm{HO}_{2} \rightarrow \mathrm{H}_{2} \mathrm{O}+\mathrm{O} \\
\text { Whitten et al }(1973)\end{array}$ & $K_{27}=\exp (-500 / T)$ \\
\hline $\boldsymbol{R}_{\mathbf{2 8}}$ & $\begin{array}{l}\mathbf{H}+\mathbf{H}+\mathbf{M} \rightarrow \mathbf{H}_{2}+\mathbf{M} \\
\text { Trainor et al }(1973)\end{array}$ & $K_{28}=2 \times 10^{-32} \times(273 / T)^{0.81}$ \\
\hline$R_{29 e}$ & $\begin{array}{l}\mathrm{H}+\mathrm{HO}_{2} \rightarrow \mathrm{H}_{2}+\mathrm{O}_{2} \\
\text { Gattinger (1971) }\end{array}$ & $K_{29}=1 \times 10^{-11}$ \\
\hline$R_{29 b}$ & $\begin{array}{l}\mathrm{H}+\mathrm{HO}_{2} \rightarrow 2 \mathrm{OH} \\
\text { Whittens et al (1973) }\end{array}$ & $K_{29 b}=2.8 \times 10^{-10} \exp (1000 / T)$ \\
\hline $\boldsymbol{R}_{\mathbf{3 0}}$ & $\begin{array}{l}\mathrm{H}+\mathrm{OH} \rightarrow \mathrm{H}_{2} \mathrm{O} \\
\text { Baulch et al }(1972)\end{array}$ & $K_{30}=1.4 \times 10^{-14} \times \mathrm{T} \exp (-3500 / \mathrm{T})$ \\
\hline $\boldsymbol{R}_{31}$ & $\begin{array}{l}\mathrm{OH}+\mathrm{H}_{2} \mathrm{O}_{2} \rightarrow \mathrm{H}_{2} \mathrm{O}+\mathrm{HO}_{2} \\
\text { Nicolet and Pettermans (1973) }\end{array}$ & $K_{31}=4 \times 10^{-13} \exp (-600 / T)$ \\
\hline $\boldsymbol{R}_{32}$ & $\begin{array}{l}\mathrm{H}_{2} \mathrm{O}_{2}+\mathrm{O} \rightarrow \mathrm{H}_{2} \mathrm{O}+\mathrm{O}_{2} \\
\text { Gattinger }(1971)\end{array}$ & $K_{32}=1.5 \times 10^{-13} \exp (-3000 / \mathrm{T})$ \\
\hline $\boldsymbol{R}_{33}$ & $\begin{array}{l}\mathrm{OH}+\mathrm{HO}_{2} \rightarrow \mathrm{H}_{2} \mathrm{O}+\mathrm{O}_{2} \\
\text { Hochanadel et al }(1972)\end{array}$ & $K_{33}=2 \times 10^{-10}$ \\
\hline$R_{34}$ & $\begin{array}{l}\mathrm{H}+\mathrm{H}_{2} \mathrm{O}_{2} \rightarrow \mathrm{H}_{2}+\mathrm{HO}_{2} \\
\text { Gattinger (1971) }\end{array}$ & $K_{34}=1 \times 10^{-13}$ \\
\hline $\boldsymbol{R}_{35}$ & $\begin{array}{l}\mathrm{H}+\mathrm{O} \rightarrow \mathrm{H}+\mathrm{OH} \\
\text { Scholfield (1967) }\end{array}$ & $K_{35}=2.1 \times 10^{-11} \exp (-9400 / \mathrm{RT})$ \\
\hline$R_{36}$ & $\begin{array}{l}\mathrm{H}_{2}+\mathrm{O}\left({ }^{\mathrm{I}} \mathrm{D}\right) \rightarrow \mathrm{H}+\mathrm{OH} \\
\text { Donoven and Husain }(1970)\end{array}$ & $K_{36}=1.9 \times 10^{-10}$ \\
\hline $\boldsymbol{R}_{\mathbf{3 7}}$ & $\begin{array}{l}\mathrm{H}_{2}+\mathrm{OH} \rightarrow \mathrm{H}+\mathrm{H}_{2} \mathrm{O} \\
\text { Westenburg and De Haas (1973) }\end{array}$ & $K_{37}=6.8 \times 10^{-14} \exp (-650 / \mathrm{T}) 150 \mathrm{~T} 300 \mathrm{~K}$ \\
\hline $\boldsymbol{R}_{\mathbf{3 8}}$ & $\begin{array}{l}\mathrm{OH}+\mathrm{OH}+\mathrm{M} \rightarrow \mathrm{H}_{2} \mathrm{O}+\mathrm{M} \\
\text { Wilson (1972) }\end{array}$ & $K_{38}=3 \times 10^{-30}$ \\
\hline $\boldsymbol{R}_{39}$ & $\begin{array}{l}\mathrm{H}+\mathrm{OH}+\mathrm{M} \rightarrow \mathrm{H}_{2} \mathrm{O}+\mathrm{M} \\
\text { Boulch et al }(1972)\end{array}$ & $K_{39}=6.1 \times 10^{-26} \mathrm{~T}^{-2}$ \\
\hline $\boldsymbol{R}_{\mathbf{4 0}}$ & $\begin{array}{l}\mathrm{OH}^{*} \rightarrow \mathrm{OH}+h v \\
\text { Fukuyama }(1974)\end{array}$ & $K_{40}=15 \cdot 2$ \\
\hline $\boldsymbol{R}_{\mathbf{4 1}}$ & $\begin{array}{l}\mathrm{OH}^{*}+\mathrm{O}_{2} \rightarrow \mathrm{OH}+\mathrm{O}_{2} \\
\text { Workley et al }(1972)\end{array}$ & $K_{41}=1.0 \times 10^{-14}$ \\
\hline $\boldsymbol{R}_{\mathbf{4 2}}$ & $\begin{array}{l}\mathrm{H}+\mathrm{O}_{2}^{*} \rightarrow \mathrm{OH}^{*}+\mathrm{O} \\
\text { Komova }(1973)\end{array}$ & $K_{42}=4.25 \times 10^{-12}$ \\
\hline$R_{43}$ & $\begin{array}{l}\mathrm{OH}^{*}+\mathrm{OH}^{*} \rightarrow \mathrm{H}_{2} \mathrm{O}+\mathrm{O} \\
\text { Kaufman }(1969)\end{array}$ & $K_{43}=3 \times 10^{-11}$ \\
\hline $\boldsymbol{R}_{44}$ & $\begin{array}{l}\mathrm{O}\left({ }^{1} \mathrm{D}\right)+\mathrm{O}_{3} \rightarrow 2 \mathrm{O}_{2} \\
\text { Fitzsimmons and Bair (1964) }\end{array}$ & $K_{44}=10^{-11}$ \\
\hline$R_{45}$ & $\begin{array}{l}\mathrm{O}\left({ }^{1} \mathrm{~S}\right)+\mathrm{O} \rightarrow \mathrm{O}+\mathrm{O} \\
\text { Felder and Young (1972) }\end{array}$ & $K_{45}=7.5 \times 10^{-12}$ \\
\hline$R_{46}$ & $\begin{array}{l}\mathrm{H}+\mathrm{OH}+\mathrm{M} \rightarrow \mathrm{HO}_{2}+\mathrm{M} \\
\text { Thomas and Bowman (1972) }\end{array}$ & $K_{46}=1.4 \times 10^{-31}$ \\
\hline $\boldsymbol{R}_{47}$ & $\begin{array}{l}\mathrm{H}+\mathrm{O}^{3} \mathrm{P}+\mathrm{M} \rightarrow \mathrm{OH}+\mathrm{M} \\
\text { Bates and Nicolet }(1950)\end{array}$ & $K_{47}=8 \times 10^{-33}$ \\
\hline$R_{49}$ & $\begin{array}{l}\mathrm{OH}^{*}+\mathrm{N}_{2} \rightarrow \mathrm{OH}+\mathrm{N}_{2} \\
\text { Worley et al (1972) }\end{array}$ & $K_{49}=3.6 \times 10^{-15}$ \\
\hline$R_{\text {s0 }}$ & $\begin{array}{l}\mathrm{OH}+\mathrm{O}\left({ }^{1} \mathrm{~S}\right) \rightarrow 2 \mathrm{OH} \\
\text { Filseth et al }(1970)\end{array}$ & $K_{50}=(71 \pm 3.5) \times 10^{-11}$ \\
\hline
\end{tabular}

Note: (i) fol three-body mechanism units are $\mathrm{cm}^{-6} \mathrm{sec}^{-1}$ and (ii) for two-body mechanism units are $\mathrm{cm}^{-3}$ $\sec ^{-1}$. 
Table 1(b). Photodissociation processes.


Figure 1. Eddy diffusion coefficient $\mathrm{cm}^{2} / \mathrm{sec}$.

\subsection{Computational technique}

We aim to compute the diurnal variation of ozone concentration. This diurnal variation can be obtained only after an equilibrium or quasi equilibrium value (i.e. $d y / d t \rightarrow 0$ ) is obtained by fixing all parameters. In this study it was a most challenging and computer time consuming operation to obtain the condition $\mathrm{d} y / \mathrm{d} t \rightarrow 0$. In programme, we replace space derivatives by semi-implicit finite difference method, whereas time 
derivatives are obtained by iteration method. This procedure involves two opposing constraints on the choice of the time step value: (i) To obtain the equilibrium value sufficiently rapidly, as well as to maintain stability and minimum expected accuracy time step should be as small as possible. (ii) To obtain the complete diurnal variation the time step should be as large as possible to economise on computer time. The following parametrization and mathematical technique is adopted to circumvent the above mentioned problems. Notations $L_{1}$ to $L_{6}$ and $M_{1}$ and $M_{2}$ are used to avoid complexity;

$$
\left.\begin{array}{l}
L_{1}=E(z-1)+D_{j}(t, z-1) ; L_{2}=E(z)+D_{j}(t, z) \\
L_{3}=E(z+1)+D_{j}(t, z+1) ; L_{4}=\frac{E(z-1)}{H_{a}(z-1)}+\frac{D_{j}(t, z-1)}{H_{j}(z-1)} \\
L_{5}=\frac{E(z)}{H_{a}(z)}+\frac{D_{j}(t, z)}{H_{j}(z)} ; \quad L_{6}=\frac{E(z+1)}{H_{a}(z+1)}+\frac{D_{j}(t, z+1)}{H_{j}(z+1)}
\end{array}\right\}
$$

we further write,

$$
\begin{aligned}
M_{1}= & \frac{\left(L_{1}+L_{2}\right)}{4 \Delta z}\left[\left(\frac{1}{Y_{j}(z-1, t)}+\frac{1}{Y_{j}(z, t)}\right)\left(Y_{j}(z, t)-Y_{j}(z-1, t)\right)\right. \\
& \left.+\left(\frac{1}{T(z)}+\frac{1}{T(z-1)}\right)(T(z)-T(z-1))\right]+\frac{1}{2}\left(L_{4}+L_{5}\right), \\
M_{2}= & \frac{\left(L_{2}+L_{3}\right)}{4 \Delta z}\left[\left(\frac{1}{Y_{j}(z, t)}+\frac{1}{Y_{j}(z+1, t)}\right)\right. \\
& \times\left(Y_{j}(z+1, t)-Y_{j}(z, t)\right)+\left(\frac{1}{T(z+1)}+\frac{1}{T(z)}\right) \\
& \times(T(z+1)-T(z))]+\frac{1}{2}\left(L_{5}+L_{6}\right)
\end{aligned}
$$

This new notation can be combined so that,

$$
\begin{aligned}
Y_{j}(z, t)= & \frac{\sigma}{2 \Delta z}\left\{M_{2}\left[Y_{j}(z, t)+Y_{j}(z+1, t)\right]-M_{1}\left[Y_{j}(z, t)+Y_{j}(z-1, t)\right]\right\} \\
& +P_{j}(z, t)+Q_{j}(z, t) \cdot Y_{j}(z, t)
\end{aligned}
$$

( $\sigma=$ filter for the constant error).

This time derivative is however considered by the predictor-corrector method by writing:

$$
Y_{j}^{*}(z, t+1)=Y_{j}(z, t)+\Delta t \times \Delta Y_{j}(z, t),
$$

where $Y_{j}^{*}$ is a predicted value, which is further corrected as

$$
Y_{j}(z, t+1)=Y_{j}^{*}(z, t+1)+(\Delta t / 2)\left\{\Delta Y_{j}(z, t)+Y_{j}^{*}(z, t+1)\right\} .
$$

Equation (1) is therefore numerically solved (CDC 3600 and DEC 10 computer) using (8) to $(10)$ for space and time parts respectively.

After considerable trial and errors, we could fix boundary conditions, initial values 
and numerical smoothening coefficients used in (10) for obtaining stable and reasonable solutions. Initial conditions at $t=0$ are defined as

$$
Y_{j}(z, 0)=f_{j}(z) \text { where } 50<z<150
$$

for all values of $z$. In this equation $f_{j}(z)$ are known functions. Next lower and upper boundaries are defined as

$$
\begin{aligned}
& Y_{j}(z=50, t)=a_{j}(t) \\
& Y_{j}(z=150, t)=b_{j}(t), \quad t \geqslant 0
\end{aligned}
$$

where $a_{j}(t)$ and $b_{j}(t)$ are obtained from photochemical and diffusive equilibrium respectively. The coefficients $a_{j}(t)$ in (12) are fixed using photochemical equilibrium and mixing ratios of each constituents. As against this $b_{j}(t)$ are determined using conditions for diffusive equilibrium. The functions $f_{j}(z)$, which determine initial values at time $t=0$, are computed by successive interactions between oxygen atmosphere and oxygen-hydrogen atmosphere. Moreover the numerical values of smoothening coefficients are selected after considerable trials for stability of solutions. Lastly, the stability considerations were mainly determined not from rigorous mathematical arguments but by appropriateness of physical situations. We could obtain solutions over the entire domain of $z$ and $t$ with steps as large as $\left(10^{5} \mathrm{~cm}\right)$ and $5 \mathrm{sec}$, whereas in initial trials we have started from steps as small as $0.01 \mathrm{~km}$ and $0.1 \mathrm{sec}$ only.

In computer program check points have been put at many places to take care of mathematical errors which were never allowed to exceed the "change" which occurred due to physical and chemical processes during the flow pattern. Initially the solar zenith angle was fixed (i.e. overhead sun) and once the equilibrium or quasiequilibrium stage was obtained, solar zenith angle was allowed to vary to obtain temporal variation of neutral minor constituents.

\section{Results and discussion}

\subsection{Photochemical model of $\mathrm{O}_{3}$}

Ozone is a highly chemically active species because of its lowest bond strength (1.04 eV). It readily undergoes reactions which are highly exothermic. This chemical energy appears in products like $\mathrm{O}_{2}\left({ }^{1} \Delta_{9}\right), \mathrm{OH}^{*}(V \leqslant 9), \mathrm{O}\left({ }^{1} \mathrm{D}\right), \mathrm{NaO}^{*}$ etc., either as kinetic or as internal energy. Therefore, $\mathrm{O}_{3}$ plays a crucial role in the photochemistry of the upper atmosphere. The equation for $\mathrm{O}_{3}$ from table 1 can be written as:

$$
\begin{aligned}
\frac{\mathrm{d}\left(\mathrm{O}_{3}\right)}{\mathrm{d} t}= & K_{2}\left[\mathrm{O}_{2}\right][\mathrm{O}][\mathrm{M}]-K_{3}[\mathrm{O}]\left[\mathrm{O}_{3}\right]-K_{20}[\mathrm{H}]\left[\mathrm{O}_{3}\right] \\
& -J_{3}\left[\mathrm{O}_{3}\right]-J_{4}\left[\mathrm{O}_{3}\right]-K_{24}[\mathrm{OH}]\left[\mathrm{O}_{3}\right] \\
& -K_{25}\left[\mathrm{HO}_{2}\right]\left[\mathrm{O}_{3}\right]
\end{aligned}
$$

where the square bracket represents the concentration of the corresponding constituent.

There exists only a single important production mechanism:

$$
R 2: \mathrm{O}+\mathrm{O}_{2}+\mathrm{M} \rightarrow \mathrm{O}_{3}+\mathrm{M} ; \mathrm{H}=-100 \mathrm{~kJ}
$$


Because of its chemical activity ozone interacts with several species and hence there are several sink terms appearing in this model. We have therefore, examined the respective contribution of each reaction and selected only a few important ones for simplicity. There are thus four principal loss mechanisms besides photodissociation viz $R_{3}, R_{20}$, $R_{24}$ and $R_{25}$ (figure 2).

Hunt $(1971,1973)$ and Battaner (1975) neglected the reactions $R_{24}$ and $R_{25}$. However, we have found that these are of considerable significance below $80 \mathrm{~km}$ during night time. This may be because atomic oxygen and atomic hydrogen decrease sharply below $80 \mathrm{~km}$ as shown in figures $3 \mathrm{a}$, b.

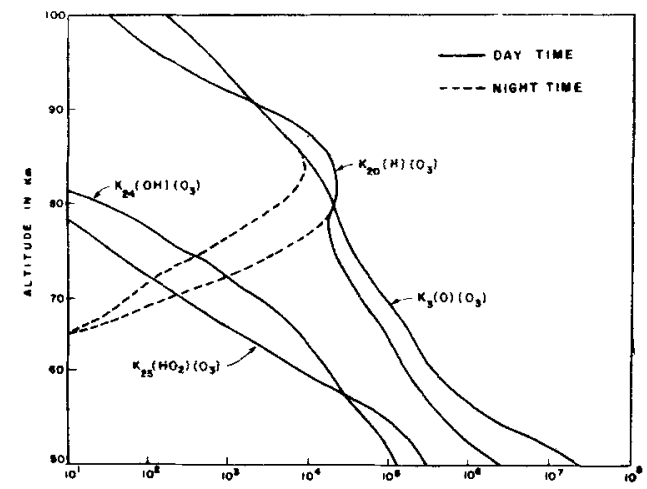

Figure 2. Reaction rate $\left(\mathrm{mol} / \mathrm{cm}^{3} / \mathrm{sec}\right)$.
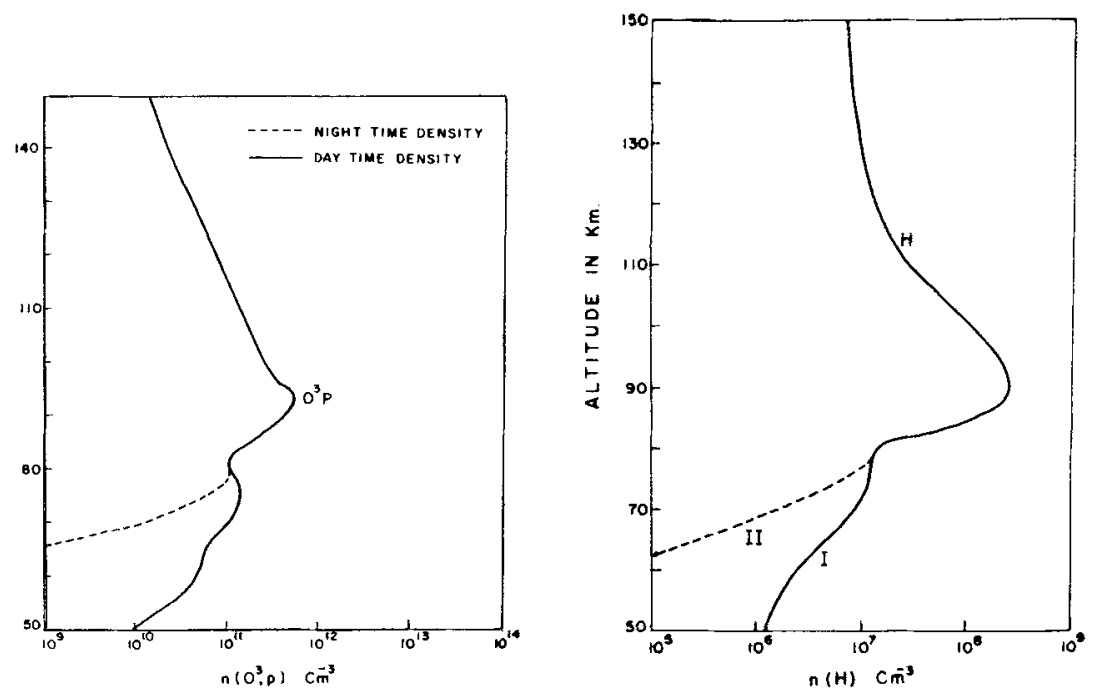

Figure 3. a. Ground state atomic oxygen concentration. b. Concentration of atomic hydrogen: Curve I is for day-time concentration and curve II is for night-time concentration. 


\subsection{Altitude and temporal variation}

Figure 4 shows altitude profiles of $\mathrm{O}_{3}$ during night and day. It shows a smaller peak at about $85 \mathrm{~km}$. Ozone plays a central role in the airglow emission of the $\mathrm{O}_{2}\left({ }^{1} \Delta_{g}\right), \mathrm{OH}^{*}$ and $\mathrm{Na}\left({ }^{2} \mathrm{P}\right)$. It is well established both theoretically and experimentally that peak emission of the above constituents appear around $85 \mathrm{~km}$. Above $90 \mathrm{~km}$ concentration of $\mathrm{O}_{3}$ decreases rapidly. This can be interpreted as the total concentration $(\mathrm{M})$ and the concentration of $\mathrm{O}_{2}$ are not sufficient to maintain the fast rate of three body mechanism $\boldsymbol{R}_{\mathbf{2}}$. It also appears that the concentration of ozone is greater during night than during day around $85 \mathrm{~km}$. This may be because during night atomic oxygen get transformed in to $\mathrm{O}_{3}$ through reaction $R_{2}$.

Further as mentioned earlier during night and below $80 \mathrm{~km}$ reactions $R_{24}$ and $R_{25}$ are important among loss reactions. These reactions need not be included during day time computation. We get reasonable accurate values of $\mathrm{O}_{3}$ concentrations within the range of 55 to $80 \mathrm{~km}$, from its photochemical equilibrium model as given below:

$$
\left(\mathrm{O}_{3}\right) \text { day }=\frac{K_{2}[\mathrm{O}]\left[\mathrm{O}_{2}\right][\mathrm{M}]}{J_{3}+J_{4}+K_{3}[\mathrm{O}]+K_{20}[\mathrm{H}]},
$$

and

$$
\left(\mathrm{O}_{3}\right) \text { night }=\frac{K_{2}[\mathrm{O}]\left[\mathrm{O}_{2}\right][\mathrm{M}]}{K_{3}[\mathrm{O}]+K_{20}[\mathrm{H}]+K_{24}[\mathrm{OH}]+K_{25}\left[\mathrm{HO}_{2}\right]} \text {. }
$$

The results based on (15), (16) and (3) are given in table 2 to illustrate this point. In figure

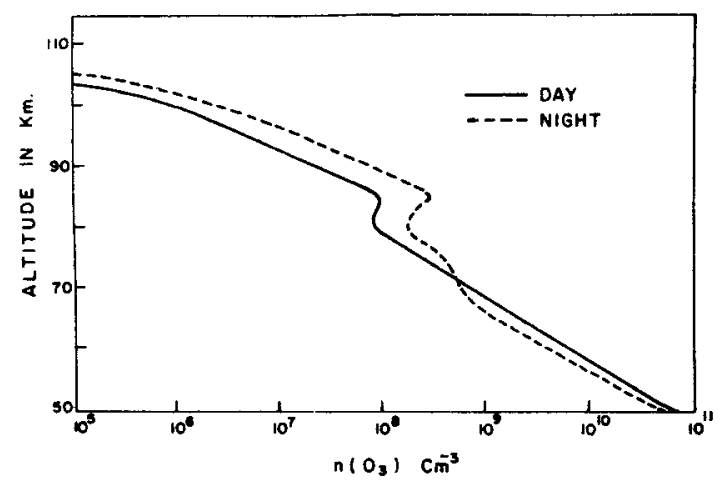

Figure 4. Daytime and nighttime concentration of ózone.

Table 2. Comparison of the results obtained from equilibrium and nonequilibrium model.

\begin{tabular}{lccccc}
\hline \multirow{2}{*}{$\begin{array}{l}\text { Altitude } \\
(\mathrm{km})\end{array}$} & \multicolumn{2}{c}{$\left(\mathrm{O}_{3}\right)_{\text {night }}\left(\mathrm{cm}^{-3}\right)$} & & \multicolumn{2}{c}{$\left(\mathrm{O}_{3}\right)_{\text {day }}\left(\mathrm{cm}^{-3}\right)$} \\
\cline { 2 - 3 } \cline { 5 - 6 } & Equilibrium & Nonequilibrium & & Equilibrium & Nonequilibrium \\
\hline 55 & $1.3 \times 10^{10}$ & $1.8 \times 10^{10}$ & & $1.8 \times 10^{10}$ & $1.2 \times 10^{10}$ \\
60 & $7.7 \times 10^{9}$ & $7.5 \times 10^{9}$ & & $8.5 \times 10^{9}$ & $1 \times 10^{10}$ \\
65 & $1.5 \times 10^{9}$ & $1.1 \times 10^{9}$ & & $1.9 \times 10^{9}$ & $3 \times 10^{9}$ \\
70 & $6 \times 10^{8}$ & $8.1 \times 10^{8}$ & & $9.7 \times 10^{8}$ & $1.2 \times 10^{9}$ \\
75 & $5.3 \times 10^{8}$ & $3.1 \times 10^{8}$ & & $3.8 \times 10^{8}$ & $2 \times 10^{8}$ \\
\hline
\end{tabular}


5 we compare our results with earlier experimental and theoretical results.. Weeks et al (1972) measured the $\mathrm{O}_{3}$ value up to $80 \mathrm{~km}$ where they reported a steady decrease. In this region our results are closer to their results. Recently Llewellyn and Witt (1977) also measured $\mathrm{O}_{3}$ concentration during twilight using a combined technique of solar occultation and $1.27 \mu$ oxygen emissions. Our results agree qualitatively up to $95 \mathrm{~km}$. However, quantitative difference exists possibly due to difference in latitude and time of measurement.

Figure 6 presents the temporal variation of $\mathrm{O}_{3}$ for $24 \mathrm{hr}$ at heights $70,75,80,85,95$ and $100 \mathrm{~km}$. From this figure we see that above $90 \mathrm{~km}$ there is no significant variation in the $\mathrm{O}_{3}$ concentration, below these altitudes the temporal variation increases with decrease in height. Further during sunset and sunrise, concentration decreases suddenly and again recovers within a period of $10-20 \mathrm{~min}$. At this time (sunset/sunrise); photodissociation processes of $\mathrm{O}_{3}$ and $\mathrm{O}_{2}$ stop/start due to which a sharp decrease of $\mathrm{O}_{3}$ concentration takes place.

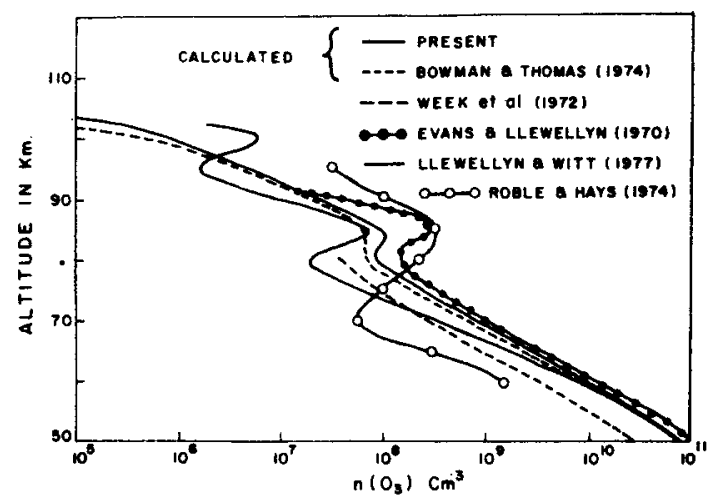

Figure 5. Comparison of $\mathrm{O}_{3}$ concentration profiles.

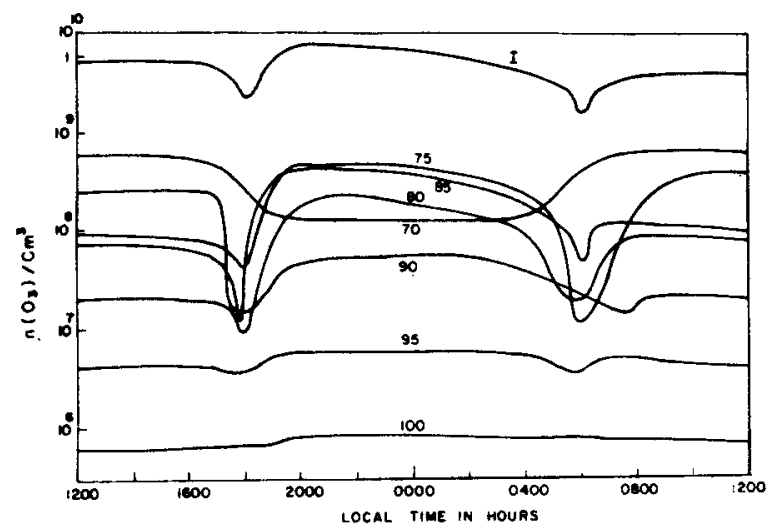

Figure 6. Diurnal variation of $\mathrm{O}_{3}$ (curve $\mathrm{I}$ is the integrated diurnal variation of $\mathrm{O}_{3}$ ). 


\section{Acknowledgement}

The author acknowledges encouragement from Dr A D Tillu of Poona University and Dr Bh V Ramanamurty, Director of the Institute. He also thanks the Computer Division staff of TIFR, Bombay for their co-operation and Mrs S P Lakade for assistance.

\section{References}

Ackerman M 1971 Mesospheric models and related experiments (ed) G Fiocco, (Dordrecht, Holland: Reidel) p. 149

Ackerman M, Biaume F and Kocharts G 1970 Planet. Space Sci. 18 p. 1639

Anderson J C and Koufman F 1973 Chem. Phys. Lett. 19483

Atkinson R and Welge K H 1972 J. Chem. Phys. 573689

Bates D R and Nicolet M 1950 J. Geophys. Res. 55301

Battaner E 1975 J. Atmos. Terr. Phys. 371155

Baulch D L, Drysdale D A and Horne D G 1970 High temperature reaction rate data, Rep. 5, Univ. of Leeds, England

Baulch D L, Drysdale D D, Horne D G and Lloyd A C 1972 Evaluated kinetic data for high temperature reactions (London: Butterworths)

Becker K H, Growth W and Schurath U 1971 Chem. Phys. Lett. 8259

Biedenkapp D, Hartshorn L G and Bair E J 1970 Chem. Phys. Lett. 5379

Chapman S 1930 Quart. J. Roy. Met. Soc. 3103

Clark I D and Wayns R P 1969 Chem. Phys. Lett. 3405

Colegrove F D, Hanson W B and Johnson F S 1965 J. Geophys. Res. 704931

Crutzen P J 1974 Can. J. Chem. 521569

Donoven R J and Hussain D 1970 Chem. Rev. 70489

Evans W F J, Hunten D M, Llewellyn E J and Vallance Jones 1968 J. Geophys. Res. 732885

Felder W and Young R A 1972 J. Chem. Phys. 566028

Filseth S V, Stuhl F and Welge K H 1970 J. Chem. Phys. 52239

Findley F D and Snelling D R $1971 \mathrm{~J}$. Chem. Phys. 55545

Fukuyama K 1974 J. Atmos. Terr. Phys. 361297

Gattinger R L 1971 The radiating atmosphere (ed) B M Mc Cormac (Dordrect, Holland: Reidel) p. 51

Gilphin R, Schiff H I and Welge K H $1971 \mathrm{~J}$. Chem. Phys. 551887

Hochanodel C J, Chormley J A and Orgen P J 1972 J. Chem. Phys. 564426

Hue R E, Herron J T and Davis D D J 1972 Phys. Chem. 762653

Hunt B G 1971 J. Atmos. Terr. Phys. 331869

Hunt B G 1973 J. Atmos. Terr. Phys. 351755

Kaufman F 1964 Ann. Geophys. 20106

Kaufman F 1969 Can. J. Chem. 471917

Komkova T G 1973 Geomagn. Aeron. 1383

Kurylo 1972 J. Phys. Chem. 763518

Uevellyn L and Witt G 1977 Planet Space Sci. 25525

Loyd K H, Low C H, McAvaney B J, Rees D and Roper R G 1972 Planet Space Sci. 20761

BNSIR Note No. C13. 458661974

Nicolet M 1970 Ann. Geophys. 26531

Nicolet and Pettermans 1973 Pure Appl. Geophys. 106-108 1400.

Schiff H I 1969 Can J. Chem. 471903

Schofield K 1967 Planet. Space Sci. 15643

Shimazaki T and Laird A R $1970 \mathrm{~J}$. Geophys. Res. $7 \$ 3221$

Shimazaki T and Laird A R 1972 J. Geophys. Res. 77276

Shimazaki T and Ogawa T 1974 J. Geophys. Res. 793411

Simonaitis $R$ and Heichlen J $1973 \mathrm{~J}$. Phys. Chem. 771932

Thomas L and Bowman M R 1972 J. Atmos. Terr. Phys. 341843 
Trainor D W, Ham D O and Kaufman F 1973 J. Chem. Phys. 584599

Wallace L and Hunten D M 1968 J. Geophys. Res. 734813

Westenberg A A and Dehaas N 1973 J. Chem. Phys. 584061

Whitten R C, Sima J S and Turco R P 1973 J. Geophys. Res. 785362

Wilson W E 1972 J. Phys. Chem. 763518

Workley S D, Coltherp R N and Potter A E 1972 J. Phys. Chem. 761511

Weeks L H, Cuikey L G and Carbin J R 1972 J. Atmos. Sci. 291138

Zipf E C 1969 Can. J. Chem. 471863 